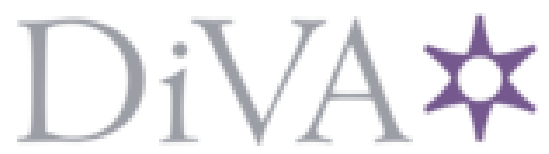

http://www.diva-portal.org

This is the published version of a paper published in Journal of Applied Physics.

Citation for the original published paper (version of record):

Davidenko, N., Pavlov, V., Chuprina, N., Davidenko, I., Bååth, L. (2006)

Thermal influence on passing of polarized light through the $\mathrm{SnO} 2$ : In2O3 layers.

Journal of Applied Physics, 100(2): 023111-1-023111-3

http://dx.doi.org/10.1063/1.2210591

Access to the published version may require subscription.

N.B. When citing this work, cite the original published paper.

Journal of Applied Physics is copyrighted by the American Institute of Physics (AIP). Redistribution of journal material is subjected to the AIP online journal license and/or AIP copyright. For more information, see: http://scitation.aip.org/content/aip/journal/jap

Permanent link to this version:

http://urn.kb.se/resolve?urn=urn:nbn:se:hh:diva-1836 


\title{
Thermal influence on passing of polarized light through the $\mathrm{SnO}_{2}: \ln _{2} \mathrm{O}_{3}$ layers
}

\author{
N. A. Davidenko, V. A. Pavlov, N. G. Chuprina, and I. I. Davidenko ${ }^{\text {a) }}$ \\ Kiev Taras Shevchenko National University, Volodymyrska Strasse 60, 01033 Kiev-33, Ukraine
}

L. Baath

Agellis Group AB, 22100 Lund, Sweden

(Received 2 March 2006; accepted 1 May 2006; published online 24 July 2006)

\begin{abstract}
Thermal dependent changes of light depolarization degree were observed in the $\mathrm{SnO}_{2}: \mathrm{In}_{2} \mathrm{O}_{3}$ (ITO) layers deposited onto the flat glass substrates which are used usually as electrodes for optoelectronic devices. The observed effect is reversible. It can be attributed to the changes of nanostructure geometry in the bulk of the ITO layer as well as on its surface. Such geometric changes involve dispersion of polarized light. The investigated effect should be taken into consideration when developing optoelectronic devices because it can provoke distortion of the optical information field. () 2006 American Institute of Physics. [DOI: 10.1063/1.2210591]
\end{abstract}

\section{INTRODUCTION}

$\mathrm{SnO}_{2}: \mathrm{In}_{2} \mathrm{O}_{3}$ (ITO) layers deposited onto the glass or lavsan substrates are usually used as the electrodes in liquid crystal and electroluminescent displays, in a photoelectric transformer of solar energy, and in optoelectronic photorefractive and holographic recording devices. ${ }^{1-5}$ A laser beam passes through the substrate with an ITO layer, while a hologram is recorded on holographic recording medium (HRM) or reconstructed. ${ }^{1}$ The basic interest in HRM consists in the absence of its influence on the information field which is formed by a coherent and linearly polarized laser beam. However, in spite of wide employment of ITO layers, these can introduce some disturbances in depicted information field due to their depolarizing properties peculiar to phaseinhomogeneous layers (PIL). ${ }^{6,7}$

\section{EXPERIMENTS}

Flat glass substrates $(50 \times 40 \mathrm{~mm})$ with thicknesses of $1.2,2.2$, and $3.2 \mathrm{~mm}$ with deposited ITO layers were used as samples for investigation. Silver electric contacts were deposited along the narrow edge of the substrate onto the surface of an ITO layer for current pulse input. The parameters of these pulses corresponded to erasing pulses used in holographic recording on the photothermoplastic medium. ITO layers with thicknesses of 700-3000 $\AA$ and surface resistances of $10-50 \Omega / \square$ were deposited by magnetron and thermal evaporations. During the pulse duration of $90 \mathrm{~ms}$ energies of 10-110 J evolved in the ITO layer. Light intensity $(I)$ which passed through the glass substrate with the ITO layer and a polarizer $\left(P_{2}\right)$ (analyzer) was measured experimentally. Either a diode laser $(5 \mathrm{~mW}, \lambda=655 \mathrm{~nm})$ or an incandescent lamp with a monochromator and a polarizer $\left(P_{1}\right)$ was employed as a light source. Light beam was directed normal to the sample surface. Light intensity $I_{0}$ reaching the sample surface was changed by the neutral light filters within the range of $1-10^{3} \mathrm{~W} / \mathrm{m}^{2}$. The dependencies of

${ }^{a)}$ Electronic mail: daviden@ukrpack.net
$I / I_{N=0}$ on time $t$ after current pulse input into the ITO layer with interval of $1 \mathrm{~s}$, on the number $N$ of current pulses, on the angle $\theta$ between the polarization planes $P_{1}$ and $P_{2}$, on light wavelength over the range of 400-900 nm were measured, where $I$ is the light intensity which passed through the sample and analyzer after current input and $I_{N=0}$ is the light intensity measured at the same conditions but at temperature $T=293 \mathrm{~K}$. The degree of polarization $\Pi=\left|\left(I_{\|}-I_{\perp}\right) /\left(I_{\|}+I_{\perp}\right)\right|$ (Ref. 8) of light which passed through the glass substrate with the ITO layer was measured over the temperature range of $290-395 \mathrm{~K}$ and over the range of light wavelength of 400-900 nm, where $I_{\|}=I_{N=0}$ for $\theta=0$ and $I_{\perp}=I_{N=0}$ for $\theta$ $=\pi / 2$. These measurements were fulfilled in the thermostat with optical windows; the sample temperature was determined by thermopair contacting with the substrate surface.

\section{RESULTS AND DISCUSSION}

Pronounced changes of light intensity $I$ under the influence of current pulses were not observed while unpolarized light passes through the investigated samples with ITO layers. Essential changes of laser light intensity as well as intensity of light from source with polarizer $P_{1}$ passing through the sample and analyzer $P_{2}$ were registered. Typical dependencies of $I / I_{N=0}$ on time $t$ for different values of $N$ and $\theta$ are shown in Fig. 1. After current pulse input into the ITO layer the intensity $I$ for $\theta=\pi / 2$ firstly decreases; next it grows and for large $N$ can reach a value by an order of magnitude greater than the initial one. For smaller angles $\theta$ the opposite character of the dependency of $I / I_{N=0}$ on time $t$ was observed. These effects are reversible: after $I$ relaxes until its initial value input of the current pulses involves the same changes of light which passed through the sample. Relaxation of the light intensity $I$ occurs exponentially with characteristic times of 200-300 s after the influence of current pulses ceases and the maximal value is reached. The characteristic time increases in the samples with a thicker glass substrate. Evidently, this tendency is caused by natural heat abstraction from the ITO layers. Changes of $I / I_{N=0}$ do not 

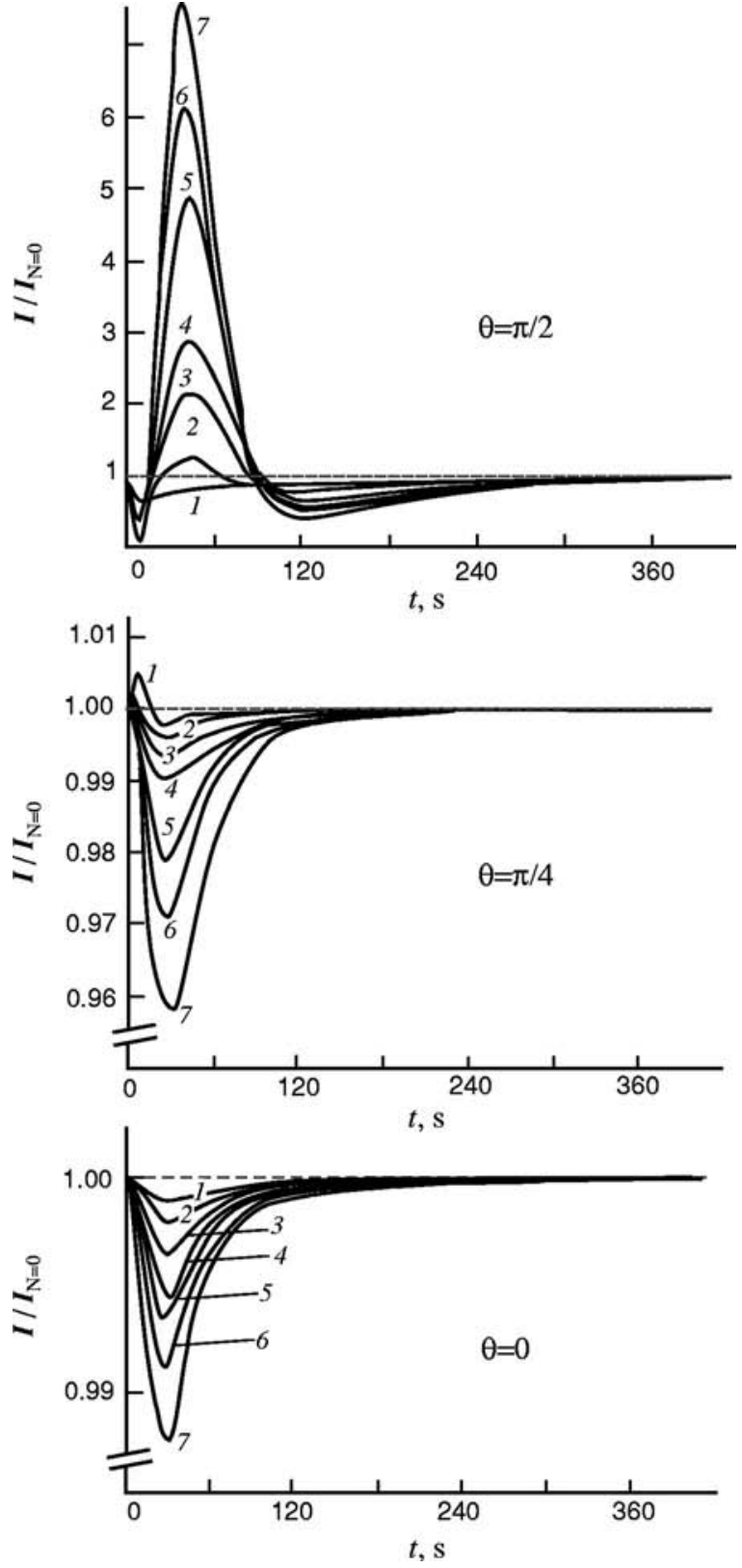

FIG. 1. Time dependencies of $I / I_{N=0}$ for different values of $\theta$ and $N=1$ (1), 3 (2), 5 (3), 10 (4), 15 (5), 20 (6), and 25 (7) $(\lambda=650 \mathrm{~nm}$, the thickness of the glass substrate is $2.2 \mathrm{~mm}$, the surface electric resistance of the ITO layer is $20 \Omega$, and the energy of heat abstraction corresponding to one current pulse is $\sim 55 \mathrm{~J}$ ).

depend on the orientation of the polarization plane of incident light relative to the current direction in the ITO layer, i.e., on the rotation of the sample plane around the direction of light propagation. The general character of the effects investigated is similar over the whole investigated ranges of $I_{0}$ and $\lambda$ in the samples with different thicknesses of the ITO layers, in the samples obtained using different technologies.

Since the depolarizing properties of the ITO layers are independent of the direction of current propagation, one can conclude that these properties are caused neither by onedimensional electrons moving while the current pulses pass nor by peculiarities of the band structure of ITO. It would be natural to suppose that observed effects of light depolarization can be attributed to the reversible structural changes in

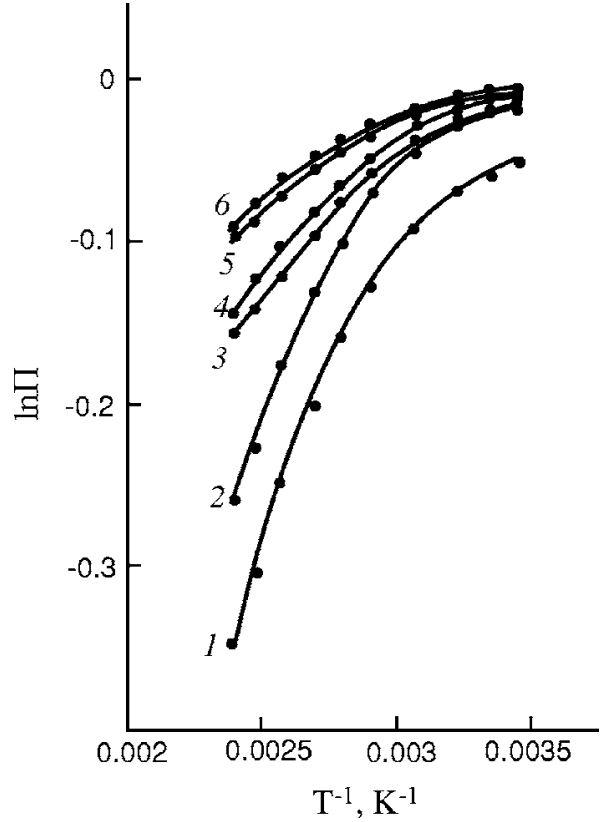

FIG. 2. Dependencies of $\ln \Pi$ on $T^{-1}$ for $\lambda=404 \mathrm{~nm}$ (1), $434 \mathrm{~nm}$ (2), $534 \mathrm{~nm}$ (3), $604 \mathrm{~nm}$ (4), $704 \mathrm{~nm}$ (5), and $804 \mathrm{~nm}$ (6) (the thickness of the glass substrate is $2.2 \mathrm{~mm}$, and the surface electric resistance of the ITO layer is $20 \Omega$ ).

the ITO layers which happen due to abstraction of additional heat. Experimental dependencies of $\ln \Pi$ on $T^{-1}$ measured using light with different wavelengths (Fig. 2) can serve as additional confirmation of the above made supposition. It should be pointed out that thermal influence on $\Pi$ for the glass substrates without ITO layers was not observed over the whole investigated range of light wavelengths, whereas changes of optical density of the same substrates with ITO layers reach $15 \%$ while $\lambda$ changes.

Atomic force microscope (AFM) image of the surface of the ITO layer is shown in Fig. 3. It is well known that optical and geometric parameters of PIL can be considered as a superposition of external (roughness) and internal (cracking, electric domains, dislocations, etc.) components. ${ }^{6,7,9}$ The observed effect of $\Pi$ decrease under $T$ growth is evidently not

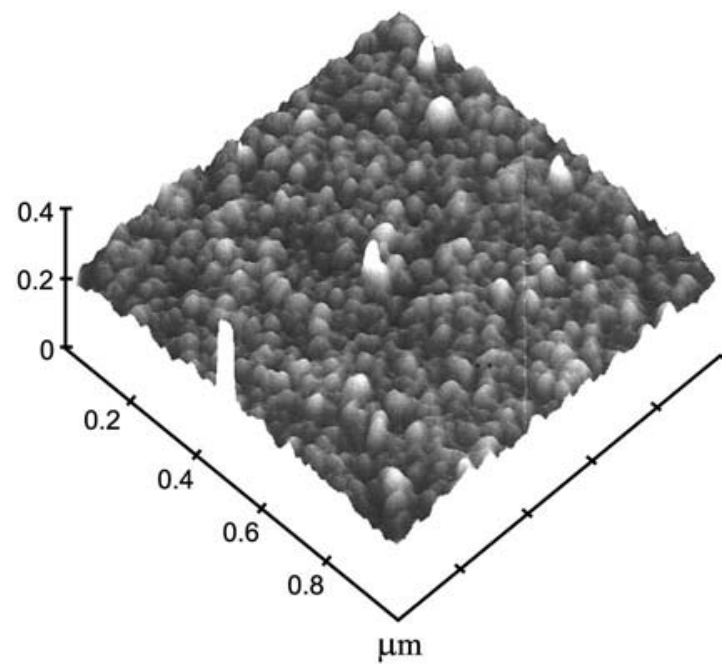

FIG. 3. AFM image of the surface of the ITO layer. 
caused by increasing concentration of monoenergetic and isometric domains or dislocations in ITO structure because dependencies of $\ln \Pi$ on $T^{-1}$ are nonlinear (Fig. 2). ${ }^{9}$ On the other hand, $\Pi$ growth as well as strengthening of its dependency on $T$ while $\lambda$ decreases (Fig. 2) testify that under temperature growth the rise of concentration of nanocenters depolarizing light happens. Such centers are mainly located on the interface of the ITO layer. ${ }^{6,7}$

Taking into account the correlation between dependencies of $I / I_{N=0}$ on $t$ (Fig. 1) and dependencies of $\Pi$ on $T$ (Fig. 2) one can conclude that light depolarization in the ITO layers occurs mainly on the surfaces. This effect reveals itself with more power while $N$ and $T$ increase. Wide employment of the ITO layers in modern optoelectronic devices requires more information about observed depolarizing properties as well as detailed investigations of their physical reasons. We consider researches on changes of nanosized surface structure of the ITO layers under influence of electric field and temperature as a following step of our activity in this field.

${ }^{1}$ N. A. Davidenko and A. A. Ishchenko, Theor. Exp. Chem. 38, 88 (2002). ${ }^{2}$ N. A. Davidenko and A. A. Ishchenko, Tech. Phys. Lett. 28, 483 (2002). ${ }^{3}$ J.-M. Nunzi, C. R. Phys. 3, 1 (2002).

${ }^{4}$ N. A. Davidenko, N. A. Derevyanko, A. A. Ishchenko, N. G. Kuvshinsky, A. V. Kulinich, O. Ya. Neilands, and M. V. Plotniece, Russ. Chem. Bull. 53, 1611 (2004).

${ }^{5}$ N. A. Davidenko et al., High Energy Chem. 39, 254 (2004).

${ }^{6}$ A. G. Ushenko, Opt. Eng. 34, 1088 (1995).

${ }^{7}$ A. G. Ushenko, Laser Phys. 10, 1143 (2000).

${ }^{8}$ M. Born and E. Wolf, Principles of Optics (Pergamon, New York, 1964). ${ }^{9}$ Ch. Kittel, Introduction to Solid State Physics (Willey, New York, 1970). 